\title{
Reseña: La revolución de las Mariposas. A diez años de La Gesta del Nombre Propio.
}

The revolution of butterflies. Ten years from The deed of the Proper Name.

Lic. María Irene Incerti

Fecha de recepción: 19 de mayo 2017

Fecha de aprobación: 20 de enero 2018

El libro "La revolución de las Mariposas. A diez años de La Gesta del Nombre Propio" es un trabajo realizado de manera colectiva, impulsado por el Ministerio Público de la Defensa de la Ciudad Autónoma de Buenos Aires junto al Bachillerato Popular Trans Mocha Celis, orientado a la población trans. En este trabajo se propone e incorpora a travestis y trans como sujetxs ${ }^{1}$ investigadorxs y no solo como objeto de estudio. Este libro parte de la información que en 2005 fue reunida en La Gesta del Nombre Propio y la completa y enriquece a partir de una nueva investigación, que amplía el alcance de sujetxs, incorporando a los varones trans. Indaga las dimensiones de salud, educación, vivienda, trabajo, el impacto de Ley de Identidad de Género, datos sociodemográficos. Y analiza a qué edad asumieron socialmente su identidad de género y como eso impacta en el acceso a otros derechos. A su vez, los datos producidos por la investigación son interpelados por la propia lectura analítica de travestis y trans y por expertos/as en la temática. Cada capítulo es acompañado por interpretaciones que arrojan luz sobre los datos de la investigación.

En el año 2005 se publicó la investigación coordinada por Lohana Berkins y Josefina Fernández, conocida como La Gesta del Nombre Propio. A poco más de diez años se retoma esta obra y se encara una nueva investigación respecto a la situación de la población trans en la Ciudad de Buenos Aires, impulsada por el Ministerio Público de la Defensa de la Ciudad Autónoma de Buenos Aires y el Bachillerato Popular Trans Mocha Celis. En esta investigación, realizada de manera participativa y en conjunto con la comunidad trans, releva datos en relación a los derechos de este colectivo.

\footnotetext{
* Autores varios. Publicación del Ministerio Público de la Defensa de la Ciudad Autónoma de Buenos Aires. Año 2017. 176 pp.

${ }^{1}$ Se utiliza la letra " $x$ " para mantener la inclusión de géneros, respetando, a su vez, el estilo de escritura del libro.
} 
Dos aspectos centrales guían esta investigación por sus distintas dimensiones: el primero es comparar la situación actual de los derechos en relación al 2005, y el segundo es analizar cuál fue el impacto (si lo hubo) de la Ley de Identidad de Géneros.

Según el análisis expuesto en esta investigación, esta ley es resultante de la investigación del 2005, e implica un giro cultural en el ámbito del derecho. Según la normativa se reconocen las vivencias y sentimientos de las personas para poder definir la identidad de género.

El libro, dividido en distintos apartados, con introducciones colectivas y análisis realizados por distintxs especialistas, va recorriendo las que consideran como exclusiones y desigualdades estructurales y persistentes. En efecto no se limitan a la desigualdad y exclusión económica, sino que consideran, a su vez, las de género, edad y nacionalidad.

Resulta necesario aclarar que, a pesar de la fuerte base en investigación cuantitativa, la misma no se limita a este aspecto. Sino que el dato duro es cuestionado y reformulado, es atravesado por las vivencias y testimonios que se exponen de integrantes de la comunidad trans, donde se visibiliza la propia voz de Ixs protagonistas, a la vez que se actualizan las realidades sociales de esta población.

A lo largo de esta investigación, y cruzando las distintas aristas, se identifican dos tipos de injusticia: la socioeconómica y la cultural o simbólica. Estas, a su vez, se ven ligadas y conectadas; están atravesadas por una acumulación de desventajas y derechos vulnerados que son explicitados en los distintos pasajes del libro. Es así que se identifica a la primera infancia, especialmente en las niñas trans, como el primer lugar de rechazo, que desencadena una serie de vulneración de derechos, como la carencia económica, el alejamiento del hogar y el abandono escolar.

Según las estadísticas que se muestran en los resultados de esta investigación, cerca del $70 \%$ de las mujeres trans tuvieron que abandonar el hogar familiar aún siendo menores de edad. Este dato, cuando se contrapone a los datos de Ixs jóvenes de la Ciudad de Buenos Aires en general, marcan una brecha de desigualdad importante. Por otra parte, este alejamiento del seno familiar está vinculado a la asunción de la identidad de género.

Esta vulnerabilidad suele estar acompañada por el abandono escolar temprano, lo cual afecta indiscutiblemente las posibilidades de acceso a empleos formales (según las estadísticas que se presenta, son muy pocas las personas de esta comunidad que logran terminar los estudios secundarios). A estos datos se asocia el hecho de que la mayoría de las mujeres trans/ travestis se encuentra en situación de prostitución, a pesar de que este dato es menor respecto al expresado en 2005. Aún es un porcentaje que se mantiene alto y con una edad baja de ingreso a la prostitución (asociado con la salida del hogar familiar y la necesidad de auto-sostén). La mayoría de estas mujeres expresa intenciones de 
abandonar la actividad si pudiera. A su vez esto se encuentra relacionado con nivel educativo alcanzado. Por lo cual hay una vinculación directa entre el alejamiento del hogar familiar, el abandono de los estudios y el inicio en la prostitución como actividad laboral. A la inversa, la investigación sostiene que a mayor nivel educativo, mayores posibilidades de obtener un empleo formal.

A diferencia de los resultados de 2005, comienzan a aparecer personas pertenecientes a esta comunidad con acceso a empleos formales, los cuales están vinculados a políticas focalizadas asociadas a tales fines. Si bien el número es bajo, comienza a dar cuenta de la importancia de las políticas destinadas a esta población. En los resultados de esta investigación se resalta la importancia de las políticas vinculadas a finalización de estudios, y la formación laboral. También se destacó la importancia de la Ley Provincial de Cupo Laboral Trans (Provincia de Buenos Aires, 2015) y la necesidad de que se repliquen legislaciones similares en otras provincias, como que se garantice su cumplimiento.

Otro de los aspectos que se destacan en la investigación es el vinculado al acceso a la salud. Si bien el acceso sigue siendo limitado, se expresaron mejoras respecto al 2005. También se notaron diferencias, ya que los varones trans tienen menos controles preventivos en salud que las mujeres trans. Sí se aprecia que, luego de la sanción de la Ley de Identidad de Género, hay aumentos en el acceso al sistema de salud para el tratamiento hormonal, dejando la automedicación. Lo que revelan los resultados de la investigación es una preocupación por la ausencia de una perspectiva integral de la salud, y una asignación presupuestaria para garantizar los derechos reconocidos.

Por otra parte, se menciona la ausencia de mejorías en lo vinculado a la institución policial. A pesar de la sanción de la Ley de Identidad de Género, las encuestadas no encontraban diferencias sustantivas en el trato policial. Tampoco en lo vinculado al acceso a la vivienda, donde las posibilidades de acceder a alquileres vía inmobiliaria o la casa propia se ven coartados por la falta de recibos de sueldo y/ o garantías propias, sumado a la discriminación social.

Un aspecto en el que se vio un cambio notorio fue en la participación social y política del colectivo trans luego de la sanción de la Ley. Según lo expresado en esta investigación hay un aumento considerable, no solo desde el 2005, sino específicamente desde el 2012, en particular en mujeres trans. Esto es vinculado a la visibilización y reconocimiento del colectivo como tal.

Esta investigación, que aporta datos y conocimientos sobre las historias de vida del colectivo trans en la Ciudad de Buenos Aires, es un aporte sustancial para actualizar el estado de situación de esta población, y ser puntapié del debate para nuevas y necesarias políticas públicas. 
Este libro, pone de manifiesto las desigualdades y exclusiones que este colectivo aún sufre, años después de de la sanción de la Ley de Identidad de Género y de las conquistas alcanzadas en los últimos años en materia de reconocimiento.

Este es un libro movilizador, crudo y realista, que continua con la lucha por el reconocimiento y garantía de derechos del colectivo trans. Se explicita la necesidad de mayores niveles de compromiso y formación en los/las diseñadores y ejecutores de las políticas públicas para que puedan implementarse nuevas intervenciones orientadas a la contención y pensadas en las especificidades que esta población requiere, para así poder garantizarle a este colectivo el pleno ejercicio de sus derechos. 